\title{
Leaders associatifs migrants et mobilité transnationale
}

\author{
Une réflexion sur les trajectoires et activités \\ des membres d'OSIM ${ }^{1}$ en France
}

\begin{abstract}
Claire Vincent-Mory²
[Résumé] Les leaders associatifs migrants constituent un objet d'étude privilégié pour la recherche sur la mobilité et le transnationalisme. Nos enquêtes révèlent cependant que leurs trajectoires ne sont pas marquées par une continuité circulatoire mais par des ruptures de mobilité et des périodes d'immobilité. La création d'associations de solidarité tournées vers le développement du pays natal permet à ces acteurs de retrouver une mobilité. Ces nouvelles circulations entrent alors dans un processus de réaffiliation vis-àvis du « pays d'origine » et participent à l'acquisition d'un capital mobilitaire. Si nos résultats confirment la relation indiscutable, dans les représentations individuelles et collectives des migrants, entre la mobilité transnationale, comme pratique sociale, et la migration, comme expérience, ils montrent que ce lien est un construit social. L'engagement associatif dans une OSIM participe à des stratégies d'ascension sociale, selon lesquelles la mobilité transnationale est l'aboutissement du parcours migratoire.
\end{abstract}

Mots-clés : Mobilité transnationale, OSIM, pays d'origine, capital mobilitaire, parcours migratoire, sédentarités.

[Abstract] The leaders of international migrant organizations are a privileged research subject in scientific literature about mobility and transnationalism. However, our fieldwork reveals that their trajectories are rather characterized by breaks and immobility than continuous movements across borders. The creation of transnational development organizations for hometown communities initiates a new kind of mobility. These transnational movements take part in a process of reaffiliation to the "country of origin" and both indicate and contribute to the acquisition of mobile capital. While our results confirm the undisputable link between transnational mobility as social practice, and migration as experience, they also show that this link is a social construct. Transnational mobility becomes the culmination of the migratory path and migrant associative commitment leads to upward social mobility.

Keywords: Transnational mobility, hometown organizations (HTOs), country of origin, mobile capital, migratory path, sedentariness.

1 Organisation de solidarité internationale issue des migrations.

2 Université Paris Ouest Nanterre La Défense - Institut d'études pour le développement économique et social, Université Paris 1 Panthéon-Sorbonne. 


\section{Introduction}

À propos des trajectoires contemporaines des migrants, Catherine Wihtol de Wenden observe que l'« installation dans la mobilité comme mode de vie a remplacé la double absence décrite par Abdelmalek Sayad par la coprésence» (Wihtol de Wenden, 2010 : 27). Cette affirmation s'inscrit dans une perspective contemporaine selon laquelle le paradigme de la mobilité (Faist, 2006 ; Weber, 2009; Urry, 2000) serait devenu le prisme privilégié pour saisir les trajectoires migrantes. Si la mobilité fait aujourd'hui figure d'évidence, elle est issue en réalité d'un glissement conceptuel progressif au cours des vingt dernières années. En effet, jusqu'au début des années 1990, le prisme de l'intégration, l'approche diachronique des trajectoires migrantes et l'idée de cycle migratoire (Bastenier, Dassetto, 1993) tendaient à établir, dans une perspective parfois évolutionniste (Tripier, 2004 ; Leclerc-Olive, 2002 : 109), l'existence d'un processus linéaire allant de la mobilité première du migrant à son immobilisation progressive dans la société d'accueil et à la modification de ses appartenances symboliques, territoriales et citoyennes 3 . Ces « épistémologies de la sédentarité » (Leclerc-Olive, 2015) ont laissé place aux analyses dynamiques, affirmant l'hypothèse d'un mouvement permanent grâce auquel les migrants déploieraient leurs activités dans plusieurs lieux à la fois, autrement dit dans des espaces sociaux transnationaux (Berthomière, Hily, 2006). La recherche privilégie désormais l'étude des flux, des circulations transnationales (Simon, 2006; Cortes, Faret, 2009), la diversité des stratégies des migrants et les expériences de co-présence (Dufoix, Guerassimoff, De Tinguy, 2010). Dès lors, comme l'affirme Serge Weber, « la circulation et la mobilité circulatoire s'imposent comme les paradigmes pertinents pour interroger les migrations aujourd'hui» (Weber, 2009: 121). Si ces paradigmes ont constitué une véritable avancée en permettant de considérer ensemble des ancrages pluriels et simultanés, ils ont conduit à essentialiser la figure du migrant en tant qu'acteur en mouvement. Ainsi,

3 De nombreux travaux sociologiques ont décrit et analysé l'évolution des approches universitaires des migrations. Ainsi, Dominique Schnapper a montré comment les perspectives en termes d'« assimilation » puis d'« intégration » ont progressivement été abandonnées, en raison de leur ambiguité fondamentale puisqu'elles appartiennent au langage à la fois politique et scientifique. À la dénonciation de l'assimilation dans les années 1980, «processus niant et détruisant les cultures d'origine des immigrés [...], connoté avec le nationalisme, le colonialisme et l'impérialisme» (Schnapper 2007: 14), a succédé la critique scientifique de l'intégration dès la fin des années 1990, «mot [...] caractéristique du point de vue de dominants, imposant leurs normes à des dominés qui pourraient se réclamer d'une double culture, sans tenir compte de leur légitime recherche d'identité » (Schnapper, 2007: 16). Néanmoins, ces changements sémantiques sont à considérer avec précaution car, en sociologie, "qu'on évoque la "régulation", I"intégration", le "faire société", le "vivre ensemble" ou le "lien social", l'interrogation reste la même, elle porte à la fois sur l'intégration des individus à la société et sur l'intégration de la société dans son ensemble » (Schnapper, 2007 : 16). 
dans un effet de bascule, la figure de l'immigré appelé à la sédentarité - signe d'assimilation - semble avoir laissé place au "transmigrant» (Tarrius, Missaoui, Qacha, 2013) : une figure de la mobilitée. Il y aurait au fond un présupposé nouveau, au sein d'une large part de la littérature scientifique, qui serait celui d'une naturalité de la mobilité. Elle se traduirait en particulier par des déplacements territoriaux et le maintien de la relation entre l'individu et sa supposée " communauté d'origine », fondée sur un sentiment d'appartenance, selon l'usage wébérien qui distingue les liens communautaires des liens sociétaux (Weber, 1971 : 41-43).

Pour les sciences sociales, plusieurs figures illustrent particulièrement ce lien entre mobilité et migration. Aux côtés des entrepreneurs ethniques (Tarrius, Missaoui, 2000 ; Ma Mung, 1999), les membres des associations transnationales de développement sont vus comme des " voyageurs longue-distance », incarnation des circulations contemporaines (Portes, Escobar, Walton Radford, 2007; Lacroix, 2016). Ces associations, que nous nommerons «Organisations de solidarité internationale issues des migrations» (OSIM) sont aujourd'hui entre deux et cinq mille en Frances. Ce sigle administratif désigne l'ensemble des associations Loi 1901 fondées par des personnes ayant elles-mêmes vécu une migration internationale et menant des actions d'aide au développement dans une région qu'elles désignent comme la « région d'origine » d'un ou plusieurs membres de leur association. La référence ambigüe à l'« origine » est issue du vocabulaire émique. Elle correspond, pour nos enquêtés, à des lieux géographiques, des collectifs et des caractéristiques attribuées ou assignées, qui participent à la formation des «nœuds de l'origine ${ }^{6}$ » (Belkacem, 2013b). Ces «nœuds» semblent centraux pour les OSIM, qui constituent aujourd'hui un mouvement associatif dy-

4 Toutes les études transnationales ne portent pas le même enthousiasme à ce sujet. En 2003, Alejandro Portes mettait en garde contre certains travaux anthropologiques qui tendraient à considérer tous les migrants comme des individus «naturellement » engagés dans des activités transfrontalières (Portes, Escobar, Walton Radford, 2003 : 876).

5 Cette estimation très large est liée au fait que les catégorisations administratives sont dans l'incapacité de rendre compte du monde des organisations transnationales de développement issues des migrations en France. Notre estimation s'est appuyée sur le Répertoire National des OSIM 2014, édité par le Forum des Organisations issues des Migrations (FORIM) et sur notre travail de terrain dans les régions Rhône-Alpes, Nord-Pas-De-Calais et lle de France.

6 "Par nœuds de l'origine, j'entends tout ce à quoi les termes "origines" et "racines" renvoient dans le vocabulaire courant : non seulement des lieux géographiques - par exemple "le pays d'origine" ou encore "le village d'origine" - mais aussi les groupes que l'on y rattache "les Maliens", "les Africains" ou encore "les Soninkés" - ainsi que les caractéristiques qu'on leur attribue - principalement exprimées en termes de "cultures", de "mentalités", d' "état d'esprit", de "traditions", de "valeurs", de "modes de vie", d"identités", de "langues" ou encore de "couleur [de peau]" » (Belkacem 2013b: 19). La mention de l'« origine » au même titre que celle des « racines » est le fruit de constructions sociales complexes (Belkacem 2013a). 
namique ${ }^{7}$ regroupant des initiatives collectives extrêmement hétérogènes, en matière de pays d'intervention, de pratiques et de profils sociologiques de leurs membres. La littérature scientifique francophone consacrée à ces associations s'est beaucoup intéressée depuis les années 1990 aux associations villageoises - petits collectifs de villageois expatriés issus des migrations de travail - actives en Afrique de l'Ouest et au Maghreb (Daum 1998; Lacroix, 2016). Pourtant, d'autres types d'associations, moins étudiés, sont actifs et numériquement importants aujourd'hui. Ainsi, de nombreuses OSIM agissent en Asie du Sud-Est, dans l'Océan Indien et majoritairement en Afrique centrale. Elles mènent des actions entre «ici » et «là-bas », impliquant de nombreux échanges matériels et symboliques, une communication soutenue et des déplacements physiques fréquents. Aussi, dans la mesure où ces OSIM construisent des espaces sociaux transnationaux, il semble nécessaire d'adopter une perspective de recherche en termes de mobilité spatiale et de pratiques circulatoires.

Notre travail d'enquête sociologique vise à questionner le présupposé de la mobilité à partir de l'expérience des leaders d'OSIM. Entre 2012 et 2014, nous avons effectué des observations de projets associatifs, ainsi que des entretiens semi-directifs auprès de cinquante-deux fondateurs, présidents ou élus de quarante-cinq OSIM ancrées dans les territoires lyonnais, lillois, parisiens et actives dans vingt-deux pays ${ }^{8}$. Dans quelles mesures les leaders des OSIM étudiées se rapprochent-ils ou s'éloignent-ils de la figure de l'individu mobile, inscrit dans des ancrages territoriaux pluriels et simultanés, et pratiquant des formes de co-présence ? Quel rapport à la mobilité entretiennent-ils ? Comment articulent-ils, dans leurs trajectoires, action associative et pratiques de mobilité ? Il semble en effet que la création d'une OSIM initie une mobilité physique nouvelle chez des acteurs dont les trajectoires semblaient plutôt marquées par des sédentarités. Ces questions en impliquent d'autres, relatives aux raisons pour lesquelles ces acteurs recherchent une mobilité par le biais de l'action associative transnationale, à des processus particuliers de reconstruction du lien aux « origines », ainsi qu'à l'acquisition d'un capital mobilitaire. Le propos de cet article est de comprendre comment et à quelles conditions ce type de leader associatif constitue une figure de la mobilité transnationale.

7Sur la période 1995-2015, pour la seule région Rhône-Alpes, nos recherches ont établi que le nombre annuel de création d'OSIM va croissant. De 3 à 4 créations en 1995-1996, les préfectures ont enregistré un nombre moyen de 10 créations annuelles de 1997 à 2002, 23 entre 2003 et 2009, pour atteindre une moyenne de 35 créations annuelles depuis 2010.

8 Bénin, Burkina Faso, Cameroun, Cap Vert, Comores, Congo Brazzaville, Côte d'Ivoire, Ghana, Guinée, Madagascar, Mali, Maroc, République Centrafricaine, République Démocratique du Congo, Togo, Sénégal ; Cambodge, Laos, Vietnam; Kosovo ; Colombie. 


\section{Sédentarités et ruptures de mobilité, en migration}

En premier lieu, notre enquête montre que les trajectoires des leaders d'OSIM sont marquées par de longues périodes d'immobilité, comme le montre le cas de Samuel Baako9. Né en 1968 dans le village d'Akuakrom au Ghana, fils de la princesse d'Akuakrom et du roi d'Asokoré, il part habiter dans le village de son père en 1974 à l'âge de 16 ans pour aller au lycée. Il entame des études supérieures puis est envoyé au Royaume-Uni pour poursuivre ses études en 1980. Une révolution éclate au Ghana à la même période et les bourses scolaires s'interrompent brutalement. Samuel rentre pour participer aux mouvements de révolte contre le gouvernement militaire. Si plusieurs de ses amis subissent des tortures et sont tués lors de ces évènements, il est épargné grâce aux pressions de son père, haut dignitaire de la monarchie Ashanti. Celui-ci obtient que son fils soit rapidement renvoyé au Royaume-Uni. La British Immigration tente alors de trouver des solutions pour les étudiants ghanéens et décide, avec l'accord de Samuel, de l'envoyer en France pour poursuivre ses études. Il vit difficilement ce qu'il considère comme une fuite, blâme le rôle joué par son père et rompt toute communication avec lui pendant quinze ans. Après quelques années, il devient entrepreneur de spectacles musicaux dans la banlieue de Lyon. Il retourne au Ghana pour la première fois en 1995 et se réconcilie officiellement avec son père. Au cours des dix années suivantes, il se rend quatre ou cinq fois dans son village natal. En 2005, il y apporte du matériel scolaire rassemblé en France. Ce geste suscite alors la colère de la reine d'Akuakrom qui considère ce don inadapté puisqu'Akuakrom n'a plus d'école... Cette réaction conduit Samuel à s'interroger sur les besoins réels de la population locale. En 2007, il est intronisé roi d'Akuakrom et crée presque immédiatement une association Loi 1901 en France, dont le nom signifie "Retour aux sources ", qu'il préside depuis. Cette association lui permet d’importer du matériel, de bénéficier de facilités douanières, de recevoir des dons et de signer des conventions avec des bailleurs. Depuis sa prise de fonction et la création de l'association, Samuel conserve sa résidence familiale à Lyon, avec sa femme et ses enfants, ainsi qu'une activité professionnelle. Mais il se rend à Akuakrom au moins deux fois par an, pour une durée totale de trois à six mois. L'association a déjà réalisé avec succès plusieurs projets de développement agro-pastoral, comme la mise en place d'une unité de transformation de produits agricoles, une filière porcine pour valoriser les déchets végétaux, ainsi que la réhabilitation de l'école primaire et la construction d'un collège ${ }^{10}$. Entre ses fonctions royales et la mise en œuvre de ces projets, Samuel est en contact quasi permanent avec ses partenaires locaux : il échange près d'une dizaine d'appels téléphoniques par jour lorsqu'il est en France.

9 Tous les noms cités ont été anonymés.

10 Trajectoire reconstituée à partir d'un entretien réalisé le 22 juin 2012, à Lyon. 
La trajectoire de ce président d'OSIM est significative et représentative de celle des autres enquêtés. Ces leaders associatifs, majoritairement masculins, ont en moyenne 60 ans aujourd'hui. Nombre d'entre eux possèdent une double nationalité (France et pays de naissance) et ont fondé une famille qui vit en France. Plus des deux tiers y ont étudié et possèdent un niveau de diplôme équivalent ou supérieur au baccalauréat ${ }^{11}$. Enfin, tous ou presque vivent dans une situation administrative et économique stable. Ces caractéristiques se retrouvent indépendamment du territoire d'intervention des associations et du pays de naissance de leurs membres. Du point de vue d'une sociologie classique des migrations, ces acteurs seraient présentés « en fin de parcours d'intégration », et leur "réussite» - fréquemment revendiquée par ceux que nous avons rencontrés - serait considérée selon le statut administratif, économique et social atteint. Parallèlement, on observe des ruptures avec le pays natal et la supposée "communauté d'origine » sur place, ainsi que des périodes d'immobilité en France pendant lesquelles les liens familiaux ou amicaux se sont distendus ou ont disparu. Dans les trajectoires de nos enquêtés, ces situations sont liées à trois types de raisons qui parfois se cumulent. Tout d'abord, des conflits politiques dans le pays de naissance ont occasionné la fermeture des frontières, désorganisé les moyens de communication et divisé la population nationale. En second lieu, les procédures administratives se sont souvent révélées problématiques, en particulier pour les personnes arrivées sur le territoire français à partir des années 1980, du fait des difficultés d'obtention des visas et titres de séjour et de la complexité des changements de statuts (notamment pour quitter la vie étudiante et entrer dans la vie professionnelle). Enfin, le coût du transport, l'obligation d'honorer le groupe familial et amical lors du retour au pays par des cadeaux de nature variée, l'importance des sollicitations financières auxquelles est confronté le migrant sur place et qu'il ne peut refuser, demandent aux individus un capital important qu'ils ne sont pas toujours en mesure de mobiliser.

Néanmoins, bien que l'ensemble des trajectoires de nos enquêtés soit marqué par des formes d'immobilité, leurs expériences sont hétérogènes. Plus précisément, on peut identifier, parmi les leaders d’OSIM, trois "générations migratoires ", qui correspondent à des caractéristiques sociologiques et des contextes socio-historiques différents. Un premier ensemble d'individus est arrivé en France entre 1954 et 1975. Il s'agit en partie d'hommes issus de milieux privilégiés, venus en France pour poursuivre des études supérieures, grâce aux dispositifs existant entre la France et ses anciennes colonies. D'autres, plus nombreux, issus du monde rural ouest-africain pour la plupart, ont bénéficié de contrats de travail avec l'industrie française. Tous, cependant, ont connu une longue période d'immobilité en France (quarante ans pour la

11 Ces proportions ont été établies à partir des données collectées au cours de notre enquête. Sur la possibilité d'établir des proportions à partir de données ethnographiques, voir Florence Weber, 1995. 
plus longue, neuf ans en moyenne) avant de retourner pour la première fois dans leur pays natal. Le second ensemble d'individus est arrivé en France entre la fin des années 1970 et la fin des années 1990. Beaucoup plus hétérogène, il se compose pour l'essentiel d'hommes et de femmes venus poursuivre leurs études supérieures et d'individus arrivés en bas âge dans le cadre des dispositifs de regroupement familial. Les individus de ce second groupe ont eux aussi connu une longue période d'immobilité en France (seize ans en moyenne) avant de retourner pour la première fois dans leur pays de naissance. Enfin, ceux arrivés depuis le début des années 2000 sont des hommes et des femmes ayant obtenu un droit d'asile ou venus poursuivre des études supérieures. Ces derniers sont les plus mobiles de notre panel et sont retournés plus régulièrement dans leur pays natal. Ces trois " générations migratoires " ne sont pas représentatives de l'ensemble des trajectoires migrantes des populations arrivées en France depuis la seconde guerre mondiale ${ }^{12}$. Cependant, elles nous renseignent sur les trajectoires de ceux qui ont choisi de fonder une OSIM.

\section{L'engagement associatif, au service de la mobilité transnationale du migrant}

On observe que la création d'une OSIM initie, pour son fondateur, une nouvelle mobilité. C'est aussi ce que montre le cas de Tep Heng et de son association. Né au début des années 1940 au Cambodge et issu d'un milieu social privilégié, il arrive en France pour poursuivre des études de médecine au début des années 1960. Médecin spécialiste, il mène une carrière de praticien hospitalier dans un grand hôpital de la région lyonnaise. Il se marie en France avec une jeune femme née au Cambodge et s'installe avec sa famille dans la banlieue lyonnaise. Il demande et obtient la nationalité française au début des années 1980. Bien qu'engagé dans une association religieuse bouddhiste et une association de médecins, regroupant toutes deux exclusivement des migrants cambodgiens et leurs enfants, Tep Heng ne rentre que deux fois dans son pays natal entre 1960 et 1970. En 1993, "après la fin d'une longue guerre », il y retourne pour la première fois et constate la pénurie dramatique de personnel, d'équipement médical et de formation à l'hôpital de Phnom Penh. De retour à Lyon, Tep Heng sensibilise des collègues médecins cambodgiens et commence à acheminer du matériel médical issu des Hospices Civils de Lyon vers des hôpitaux cambodgiens. Ces premières actions le conduisent à créer en 2001 une association avec quelques amis et collègues médecins issus de la migration cambodgienne. Puis d'autres membres, qui

12 Pour des informations précises et plus représentatives, voir les résultats de l'enquête Trajectoires et Origines menée par l'Institut national d'études démographiques (2010). Cependant, si celle-ci s'est penchée de manière approfondie sur les pratiques transnationales des acteurs issus de la migration, elle ne dit rien de la fréquence de leurs déplacements, que nous avons tenté d'évaluer dans notre travail. 
n'appartiennent pas tous au monde médical et ne sont pas d'origine cambodgienne, rejoignent cette OSIM. Tep Heng prend sa retraite en 2004. À partir de cette date, l'association mène plusieurs projets successifs : un projet hospitalo-universitaire qui articule une réhabilitation de laboratoires de recherches, l'équipement d'une bibliothèque et la mise au point d'un programme de formation assuré par des médecins français volontaires dans des hôpitaux cambodgiens; puis des projets d'adduction d'eau potable et de prévention sanitaire dans des zones rurales. Depuis la création de l'association, Tep Heng se rend au Cambodge une à trois fois par an, pour une durée totale de deux à quatre mois, afin de suivre le bon déroulement des projets ${ }^{13}$.

Ainsi, la création d'une OSIM semble marquer le début d'une mobilité qui peut prendre la forme d'une véritable co-présence. Comme le montrent les trajectoires de Tep Heng et de Samuel Baako, les trajets entre la France et le pays de naissance sont bien plus nombreux depuis le lancement de leurs activités associatives. Si ces deux cas n'ont pas vocation à résumer la diversité des contextes ou des conditions de possibilité de la création d'une OSIM en France, ils montrent que, malgré des périodes d'arrivée en France différentes, des vies professionnelles et des pays d'intervention variés, il y a, dans les deux cas, un « avant » et un " après » en termes de pratiques circulatoires. À partir de la création de leur association et de l'initiation de projets de développement, ces leaders d'OSIM voyagent régulièrement entre « ici » et « là-bas » et deviennent, de ce fait, des acteurs mobiles. On notera que, contrairement au cas idéaltypique de l'association villageoise issue de la Vallée du Fleuve Sénégal (Daum, 1998; Quiminal, 1991), les OSIM ne regroupent jamais (ou presque) uniquement des personnes nées dans le même pays, à fortiori dans la même localité. Le plus souvent le fondateur agrège autour d'un projet de développement des amis, des voisins, des collègues issus de son univers professionnel ou militant. Parmi eux, une partie seulement revendique les mêmes « origines » que celles du fondateur. Cette nouvelle mobilité se nourrit en outre d'une connectivité importante. Plusieurs présidents d'association nous ont présenté l'usage du téléphone portable et d'internet comme un véritable changement et un support essentiel dans la re-création des liens transnationaux stimulés par leurs déplacements. En effet, ces nouvelles circulations migratoires n'impliquent pas un retour définitif au pays natal, à quelques rares exceptions. Les enquêtés souhaitent plutôt vivre entre deux espaces ou davantage, comme l'explique Paul Kazadi, arrivé en France à la fin des années 1990, âgé de 35 ans au moment de notre entretien et président fondateur d'une OSIM active en République démocratique du Congo : « Moi, j’aimerais développer suffisamment les projets pour être $50 \%$ du temps là-bas, 50 \% ici. Mais pour ça, j'aimerais développer une activité professionnelle à cheval entre ici et là-bas [...]. C’est mon rêve !" ». La création d'une association

13 Trajectoire reconstituée d'après un entretien réalisé le 10 avril 2012, à Lyon.

14 Entretien réalisé le 19 septembre 2013, à Lyon. 
n'aurait donc pas vocation à préparer un retour, mais à construire une mobilité transnationale.

Dès lors, les trajectoires de nos enquêtés montrent qu'une circulation est initiée au moment où ils ont acquis une stabilité économique, sociale et administrative. Cela nous conduit à formuler plusieurs points. D'une part, comme les travaux sur la circulation migratoire et le transnationalisme l'ont démontré, la perspective selon laquelle l'éloignement géographique $\mathrm{du}$ migrant le conduirait progressivement à s'immobiliser dans le territoire d'accueil reste contredite. Ces résultats confirment que les ancrages sociaux et territoriaux, les formes d'appartenance et les pratiques citoyennes qui leur sont liées ne s'opposent pas aux pratiques transnationales (Sassen, 2009). D’ailleurs « ce sont souvent les individus immigrés les mieux établis et les plus en situation de sécurité dans la société d'accueil qui s'engagent dans de telles activités, associatives notamment» (Portes, Escobar, Walton Radford, 2003:888). D'autre part, nos résultats montrent une apparente inversion du cycle migratoire présenté par la sociologie classique des migrations : à une phase d'immobilité succède une phase de mobilité, parfois croissante, qui invalide la perspective essentialiste selon laquelle le mouvement serait la caractéristique primordiale du « migrant ».

\section{Construction du " nœud des origines " et réussite du parcours migratoire}

Si jusqu'ici la relation entre mobilité et migration semble s'inscrire, pour les acteurs étudiés, dans un processus diachronique et nuancé, il subsiste une question majeure. Pourquoi des individus issus de la migration, peu mobiles, pour certains devenus français, et qui connaissent des conditions de vie économiquement, socialement et administrativement stables, voire confortables, recherchent-ils une mobilité transnationale par le biais de l'action associative ? La réponse à cette question semble se trouver à la jonction de deux processus.

Le contexte de création des OSIM et de lancement de leurs activités fournit les premiers éléments de réponse. L’exemple de Tep Heng rejoint une situation analysée par la littérature scientifique: celle de l'arrêt de l'activité professionnelle dans le pays d'installation, qu'il s'agisse du passage à la retraite ou de la rupture d'un contrat de travail (Lacroix, Sall, Salzbrunn, 2008). Dans le cas de Samuel Baako, la décision de la Reine d'en faire son successeur et le souhait des habitants de son village natal de le voir assumer cette responsabilité politique fut le point de déclenchement. Ce contexte spécifique, peu analysé par la littérature, est revenu régulièrement dans notre enquête. Ces deux situations, pourtant différentes, constituent des moments où est particulièrement évoquée la question du rapport au «pays d'origine ». Elles mettent en avant les projets, lieux de vie et engagements des acteurs, et sont dès lors perçues comme des moments privilégiés pour (ré)affronter la question des appartenances sociales, identitaires et citoyennes. De manière générale, dans nos échanges avec des leaders associatifs d'OSIM, la distension des liens familiaux ou amicaux liés à leur " pays d'origine » fait figure de quasi-tabou. En revanche, tous présentent la relation à 
celui-ci comme souhaitable et évidente. Ainsi le président d'une OSIM intervenant en Guinée affirme :

On est directement impliqués, intéressés! On le fait pour soi-même, parce qu'on

n'a pas le droit à l'erreur. Si un truc ne marche pas, c'est dans mon village que ça ne marche pas [...]. Là par exemple, le village X. S'ils n'ont pas d'eau, c'est un peu de ma faute, je me sens un peu responsable là-dedans [...] parce que ça fait un peu sa fierté [au migrant, ndla], son honneur, de réussir ${ }^{15}$.

Ce discours, régulièrement entendu, semble entrer dans une logique de la dette qui a été largement montrée au sujet des acteurs associatifs migrants issus de la Vallée du Fleuve Sénégal ou du Maghreb (Marie, 2007 ; Daum, 1998 ; Lacroix, 2016). Or les caractéristiques sociologiques des membres des OSIM sont en partie différentes. Il semble que malgré la rupture avec le pays de départ, la nouveauté de l'engagement associatif et la diversité des modalités de mobilité (qui oscillent entre décisions individuelles et collectives), le sentiment d'être «endetté » vis-à-vis du groupe d'« origine » constitue un élément central du rapport des migrants leaders d'OSIM à leurs pays de naissance. Dans ce contexte, l'action associative transnationale en faveur du développement s'inscrit dans le registre de l'utilité et de la quête de reconnaissance. Tep Heng décrit en ces termes son engagement: "C'est une reconnaissance de [moi-même, ndla]. Au moins je suis utile, dans mon pays, et mon pays me reconnait, que s'ils ont un enfant, même s'il n'est plus avec nous, il pense à nous. ». Comme lui, la plupart des enquêtés semblent chercher à dépasser, par l'action associative, un sentiment de mise à l'écart de la part des institutions nationales dans les pays de naissance et d'installation ${ }^{16}$, et pour certains, un sentiment de distance vis-à-vis de leurs " compatriotes », « ici » et «là-bas ». Ainsi, pour l'un des membres du bureau de l'association de Tep Heng :

[ll faut] surtout enlever le complexe, soit de culpabilité, soit d'infériorité [...] des migrants eux-mêmes. Et moi, je suis contre le plaidoyer des migrants qui consiste à dire «il faut taper du poing sur la table pour qu'on nous reconnaisse ». Je suis plutôt pour l'action des migrants eux-mêmes, pour ce qu'ils font et que la reconnaissance vienne de leur action! Et non parce qu'ils vont réclamer quelque chose $e^{17}$.

Par ailleurs, au-delà de la construction de partenariats avec une ou plusieurs organisations locales - qui participe à l'établissement de relations entre membres d'OSIM et habitants du territoire d'intervention - il semble que l'action associative construise l'illusion d'une continuité relationnelle et sociale avec le "pays d'origine » et masque

15 Entretien avec Ibrahima Ba, 13 avril 2012, Saint Denis en Bugey.

16 La majeure partie de nos enquêtés possède la double nationalité. Cependant, les conditions d'exercice de leurs citoyennetés demeurent difficiles: dans plusieurs pays d'Afrique centrale notamment, le vote depuis l'étranger n'est pas possible. En outre, leur parcours migratoire les catégorise socialement comme des nationaux " pas comme les autres » et suscitent des discours et représentations extranéisants, en France comme dans leur pays de naissance. 17 Entretien avec Anoulath Pravong, 17 avril 2013, Paris. 
de longues périodes d'affaiblissement ou de rupture des liens, ainsi que différentes échelles de déplacements et de territoires d'appartenance des acteurs. Or, les discours des leaders d'OSIM rencontrés, comme ceux de leurs partenaires associatifs, s'accordent sur un point : le maintien de liens continus entre « ici » et « là-bas » est garant de la qualité des projets entrepris. Ainsi, selon un membre du bureau d'une association béninoise, partenaire d'un projet de développement agro-pastoral porté par une OSIM :

Les Béninois de l'extérieur sont souvent craintifs. Plus ils ont vécu là-bas [en France, ndla], plus ils [...] craignent de faire un projet ici. Ce qui fait la différence entre ceux qui mènent des projets et les autres, c'est s'ils ont su garder et entretenir des liens. Sinon, même s'ils veulent réaliser un projet, s'ils ne connaissent plus personne, ils ne pourront pas. Ils se feront avoir, ils ne pourront pas mobiliser les gens des localités. Plus ils vivent là-bas, plus ils développent une nouvelle mentalité. C'est normal, mais après, parfois, ils ne comprennent plus les problématiques de l'Afrique. Ils ne peuvent plus travailler avec les gens ${ }^{18}$.

Pourtant, les «Béninois de l'extérieur » avec qui il travaille n'ont aucun lien familial ou amical avec les habitants du village dans lequel est mis en place ce projet ${ }^{19}$. Ce paradoxe, que l'on retrouve dans de nombreux discours de fondateurs et de partenaires d'OSIM, conduit à penser que ces actions associatives transnationales, en voulant "prouver » l'existence de liens avec des habitants du territoire "d'origine", contribuent à la construction d'une "pensée des racines » (Belkacem, 2013b) et participent à l'illusion d'une immuabilité et d'une permanence des appartenances.

Cette entreprise de réaffiliation des leaders associatifs à leurs « pays d'origine » présente une particularité. Elle s'appuie sur leur mobilité retrouvée, qui constitue l'atout majeur du processus de performation du «nœud des origines». Les trajectoires de Samuel Baako et de Tep Heng nous permettent de saisir combien l'expérience de déplacement transfrontière est liée à la possession d'un certain nombre de capitaux. Audelà de leurs différences, ces deux acteurs possèdent des caractéristiques communes : l'un comme l'autre ont fait des études supérieures et acquis des capitaux social et économique par leur statut professionnel, ainsi que des capitaux politique et symbolique par leur héritage familial. Plus largement pour l'ensemble de nos enquêtés, il semble y avoir une corrélation entre la possession de différents capitaux, le statut social et la création associative. En ce sens, la mobilité géographique est le signe d'une stabilité administrative, mais également d'une position sociale et économique confortable, puisque les acteurs sont désormais en capacité d'assumer le coût du transport, d'aider leur entourage familial et amical et de financer des actions de développement. La circulation transnationale devient elle-même un véritable capital : les expériences

18 Entretien avec M. Franck Domingo, "premier organisateur » et membre du bureau de l'association, 29 novembre 2012, Cotonou (Bénin).

19 Le choix de la zone d'intervention s'est fait, selon les membres de I'OSIM rencontrés, grâce à une opportunité d'achat de terrain agricole. 
de mobilité accumulées par les acteurs et les réseaux sociaux développés rendent possible une appartenance multilocalisée, réelle ou virtuelle (Ceriani Sebregondi, 2007: 247-249). Ce «capital mobilitaire » est aujourd'hui particulièrement prisé par les personnes issues des migrations. Comme le montre la sociologie des mobilités, le facteur déterminant les hiérarchies sociales d'un monde globalisé est celui de la "possibilité d'interactions transfrontières et de mobilités transnationales » (Beck, 2008: 21). Il semble que l'essor de la mobilité des leaders d'OSIM rencontrés s'inscrive dans cette perspective. En ce sens, l'acquisition d'un capital mobilitaire par l'exercice d'une activité associative transnationale peut constituer une stratégie d'ascension sociale. La mobilité retrouvée signale ainsi, aux yeux de l'entourage des acteurs, «ici » et «làbas », la réussite de leur parcours migratoire.

\section{Conclusion}

L'analyse des trajectoires d'acteurs engagés dans des actions associatives transnationales de développement vis-à-vis d'un territoire qu'ils qualifient de région ou pays « d'origine » projette un nouvel éclairage sur la figure du leader associatif migrant. Les trajectoires des enquêtés ne sont pas marquées par une continuité circulatoire mais par des ruptures de mobilité. L'action associative, initiée dans un contexte croisé de stabilité administrative, sociale et économique en France et de changement de statut (professionnel ou socio-politique par exemple), conduit à développer de nouvelles mobilités transfrontalières. Elle participe également à un double processus de valorisation sociale et de reconstruction du «nœud des origines », en réinventant d'une part la relation identitaire entre ces leaders associatifs, un territoire et sa population, et en manifestant d'autre part la possession d'un capital mobilitaire. Cette analyse montre que si ces acteurs demeurent une figure de la mobilité convaincante, les relations entre mobilité et migration sont plus complexes que ce que la littérature scientifique sur les associations de solidarité issues des migrations a pu parfois laisser penser. En outre, "cette forme d'engagement est cohérente avec ce que les non-migrants (l'entourage, les États, les organisations de la société civile) pensent que les migrants devraient faire» (Lacroix, 2016: 57). Dans cette perspective, il semblerait judicieux d'interroger les pratiques, les discours et les représentations des partenaires institutionnels et associatifs de l'aide au développement («i ici » et «là-bas ») vis-à-vis des membres d'OSIM, pour comprendre comment ils participent à ce processus de réaffiliation identitaire par la mobilité associative. Ceci permettrait de saisir les effets de l'articulation entre mobilité, migration et action associative de développement sur la formation des rapports de pouvoir dans le champ de la solidarité transnationale.

\section{Bibliographie}

BASTENIER A., DASSETTO F. (1993), Immigration et espace public. La controverse de l'intégration, Paris, L'Harmattan. 
BEAUCHEMIN C., HAMEL C., SIMON P. (2010), Trajectoire et Origine, Enquête sur la diversité des populations en France, Paris, INED.

BECK U. (2008), Die Neuvermessung der Ungleichheit unter den Menschen, Frankfurt, Suhrkamp.

BELKACEM L. (2013a), "Jeunes descendants d'immigrants ouest-africains en consultations ethnocliniques : migrations en héritage et mémoire des "origines" », Revue européenne des migrations internationales, vol. $29 \mathrm{n}^{\circ} 1$, p. 69-89.

BELKACEM L. (2013b), L'« enfant perdu » et le " pays d'origine ». Construction des origines et expériences migratoires de descendants d’immigrants ouest-africains en région parisienne, Thèse de doctorat, Paris, École des hautes études en sciences sociales.

BERTHOMIÈRE W., HILY M.A. (2006), « Décrire les migrations internationales. Les expériences de la co-présence», Revue européenne des migrations internationales, vol. $22, n^{\circ} 2$, p. 67-82.

CERIANI SEBREGONDI G. (2007), "La circulation migratoire aujourd'hui, émergence d'un capital mobilitaire et d'une culture de la mobilité: Le cas des migrants marocains en Europe du Sud», in E. AUDEBERT, E. MA MUNG (dir.), Les nouveaux territoires migratoires, entre logiques globales et dynamiques locales, Bilbao, Université de Deusto, p. 243-254.

CORTĖS G., FARET L. (2009), Les circulations transnationales : Lire les turbulences migratoires contemporaines, Paris, Armand Colin.

DAUM C. (1998), Les associations de Maliens en France: migration, développement, citoyenneté, Paris, Karthala.

DUFOIX S., GUERASSIMOFF C., DE TINGUY A. (2010), Loin des yeux, près du cœur. Les États et leurs expatriés, Paris, Presses de Sciences Po.

FAIST T. (2006), «The Transnational Social Spaces of Migration», Bremen, Center on Migration, Citizenship and Development, Working paper $n^{\circ} 10$.

FAIST T. (2013), « The Mobility Turn: a New Paradigm for the Social Sciences? », Ethnic and Racial Studies, vol. 11, $\mathrm{n}^{\circ}$ 36, p. 1637-1646.

FARET L. (2003), Les territoires de la mobilité. Migrations et communautés transnationales entre le Mexique et les États-Unis, Paris, Éditions du CNRS.

LACROIX T. (2016), Hometown Transnationalism. Long-Distance Villageness among Indian Punjabis and North African Berbers, London, Palgrave Macmillan.

LACROIX T., SALL L., SALZBRUNN M. (2008), « Marocains et Sénégalais de France : permanences et évolution des relations transnationales », Revue Européenne des Migrations Internationales, vol. $24, \mathrm{n}^{\circ} 2$, p. 23-43. 
LECLERC-OLIVE M. (2015), «Au-delà des épistémologies sédentaires : Les changements urbains au miroir de l'exil », Parcours anthropologiques, vol. 10, nº 1, p. 24-45.

LECLERC-OLIVE M. (2002), «Territorialités de migrants », L'homme et la société, vol.1, $\mathrm{n}^{\circ}$ 143-144, p. 105-124.

MA MUNG E. (1999), «La dispersion comme ressource», Cultures er conflits, $n^{\circ}$ 33-34, p. 89-103.

MARIE A. (2007), "Communauté, individualisme, communautarisme: hypothèses anthropologiques sur quelques paradoxes africains », Sociologie et sociétés, vol. 39, $\mathrm{n}^{\circ}$ 2, p. 173-198.

PORTES A., ESCOBAR C., WALTON RADFORD A. (2007), «Immigrant Transnational Organizations and Development: A Comparative Study ", International Migration Review, vol. 41, n 1, p. 242-281.

PORTES A., ESCOBAR C., WALTON RADFORD A. (2003), «Conclusion: Theoretical Convergences and Empirical Evidence in the Study of Immigrant Transnationalism », International Migration Review, vol. 37, n 3, p. 874-892.

QUIMINAL, C. (1991), Gens d'ici gens d'ailleurs. Migrations soninké et transformation villageoise, Paris, Éditions Christian Bourgois.

SASSEN S. (2009), La globalisation, une sociologie, Paris, Gallimard.

SCHNAPPER D. (2007), Qu'est-ce que l'intégration ?, Paris, Gallimard.

SIMON G. (2006), «Migrations, la spatialisation du regard », Revue européenne des migrations internationales, vol. 22, $\mathrm{n}^{\circ}$ 2, p. 9-21.

TARRIUS A., MISSAOUI L. (2000), Les nouveaux cosmopolitismes : mobilités, identités, territoires, La Tour d'Aigues, Éditions de l'Aube.

TARRIUS A., MISSAOUI L., QACHA F. (2013), Transmigrants et nouveaux étrangers : hospitalités croisées entre jeunes des quartiers enclavés et nouveaux migrants internationaux, Toulouse, Presses Universitaires du Mirail.

TRIPIER M. (2004), "L’immigré, analyseur de la société (note critique)», Terrains ef travaux, vol. 2, $\mathrm{n}^{\circ}$ 7, p. 173-185.

URRY J. (2000), « Mobile Sociology », The British Journal of Sociology, vol. 51, n ${ }^{1}$, p. 185203.

WEBER F. (1995), «L'ethnographie armée par les statistiques », Enquête. En ligne, consulté le 5 novembre 2012, URL : http://enquete.revues.org/272.

WEBER M. (1971), Économie et Société, Paris, Plon.

WEBER S. (2009), «Les mobilités induites par les migrations : émergence d’un champ migratoire transnational », Méditerranée, n 113, p. 121-126. 
WIHTOL DE WENDEN C. (2010), La question migratoire au XXI siècle. Migrants, réfugiés et relations internationales, Paris, Presses de Sciences Po. 